(Aus dem physiologischen Institut der Universität Graz.)

\title{
Die Localisation psychischer Vorgänge im Gehirne.
}

\author{
Einige historisch-kritische Bemerkungen. \\ Von
}

Prof. Alexander Rollett.

Die überaus zahlreichen neuen Erscheinungen anf dem Büchermarkte bedrohen, wenn sie nicht gerade Gegenstände berühren, die man selbst im Laboratorium bearbeitet, den Fortgang solcher Arbeiten stets mit Störụngen.

Aber nicht immer kann man sich den letzteren entziehen. So ergeht es mir, wenn ich jetzt zu dem in der Ueberschrift genannten Gegenstande einige Bemerkungen zusammenstelle, mit welchen ich den Lesern vielleicht einen Gefallen erweisen kann.

Ich habe im Jahre 1882 eine Studie über Gall und dessen Verkehr mit Goethe veröffentlicht ${ }^{1}$ ).

Für diese Studie benutzte ich eine umfangreiche Literatur, die am angeführten Orte zu finden ist, unter anderen auch die Hauptwerke von $\left(\mathrm{a} a l^{2}\right)$.

Ich schilderte dort, wie Gall als Apostel seiner Lehre durch Europa zog, wie er dabei mit Goethe zusammentraf, wie der letztere als sicheren Gewinn aus diesem Verkehre einen besseren

1) Aus dem Zeitalter der Phrenologie mit besonderer Beziehung auf Goethe's Verkehr mit dem Phrenologen Gall. Deutsche Revue. Herausg. von Richard Fleis cher. Jahrg. 7. Bd. 2 S. 360.

2) Gall et Spurzheim, Recherches sur le système nerveux en général etc. Paris 1809. - Anatomie et physiolog. du syst. nerv. en général et du cerv. en particul. etc. Paris 1810-1819. vol. 4 (4) et Atlas (fol) avec Planch. C. und Gall, Sur les fonct. d. cerv. et sur celles d. chac. d. ses part. etc. vol. 6. Paris 1822-1825. (Sie befanden sich damals und befinden sich jetzt in meiner Privatbibliothek.) 
Einblick in die Anatomie des Gehirnes davontrug, während er sich über die phrenologischen Auslassungen Gall's ausdrücklich die Freiheit seiner eigenen Gedanken wahrte, Dabei nahm ich aber auch Gelegenheit, mich über den Fortschritt zu verbreiten, den Gall in der Hirnanatomie machte.

Er brach vollständig mit der lange eingebürgerten Methode der Zergliederung des Gehirns, die noch von Vesal herrührte, wobei das Gehirn wie Käse von oben her in Scheiben zerschnitten wurde, und ging den Weg, dessen Spuren im 16. Jahrhundert bei Varoli und im 17. Jahrhundert bei Willis und Vieussens zu finden sind.

Gall ging vom Rückenmarke aus. In diesem dachte er sich die graue Substanz, aus welcher alle Nerven entspringen, zu Ganglienknoten geordnet, welche den einzelnen Rückenmarksnerven entsprechen, und er suchte die Fortsetzungen der Faserzüge und Substanzen des Rückenmarkes durch das verlängerte Mark in das grosse und kleine Gehirn hinein und die Anlagerung neuer Fasermassen und ganglionärer Substanz an dieselben zu verfolgen.

So gelangte er zur Auffassung des Seh- und Streifenhügels als hinteres und vorderes Hirnganglion. Ueber die Ausstrahlung der Markmasse in die Hemisphären, die beide Hirnhälften verbindenden Faserzüge, die Ursprünge der Hirnnerven eröffnete er neue und bessere Anschauungen, als man sie früher hatte.

Sein angeführter Atlas enthält für die damalige Zeit ganz hervorragend schön gezeichnete und ebenso reproducirte Abbildungen.

Gall hatte auch eine stets bereite grosse Virtuosität, das Hirn nach seiner Art zu zergliedern. Ich zeigte dann aber auch, wie die schönen Funde Gall's in der Gehirnanatomie immer in Gefahr waren, von der Fluth seiner Phantasie ertränkt zu werden, und dass wir immer nur auf gähnende Klüfte zwischen seinem ernsten Anlauf zur Gehirnanatomie und dem Possenspiel seiner Organenlebre stossen. In der genannten Studie findet sich auch auseinander gesetzt, auf welchen Säulen heute die wissenschaftliche Erkenntniss des Satzes ruht, dass die Grosshirnrinde das Organ des Intellectes ist und dass man zur Annahme einer Projection aller Sinnesorgane und der gesammten der Willkür unterworfenen Muskeln auf die Hirnrinde durch verbindende Nervenfasern kam; dass dann die Aufstellung besonderer sensorischer und motorischer Rindenfelder folgte, welche die Erinnerungsbilder der einzelnen Sinne und die Bewegungs- 
vorstellungen für bestimmte Muskelgruppen bewahren. Eine Localisation 'der Hirnfunctionen, welche himmelweit verschieden sei von der Austheilung der Wohnplätze geistiger Anlagen und edler und unedler Triebe, wie sie Gall auf Grund unhaltbarer Speculationen vornahm. Dass aber auch die neue Localisationslehre noch angefochten sei von Vertheidigern einer einheitlichen Functionsweise aller Theile der Grosshirnrinde, die als Reaction gegen Gall's absurde Localisationen williger Aufnahme begegnete.

B roc a war, als diese meine Aeusserungen fielen, schon gewürdigt. Es lagen die Arbeiten von Meynert, Fritsch und Hitzig vor, und Ferrier, H. Munk und Exner hatten eine Reihe von Arbeiten über die Localisation schon veröffentlicht, ebenso Goltz seine gegnerischen Arbeiten bis zur 4. Abbandlung. Heute kennen wir nun auch die erst 10 Jahre später erschienene wichtige 6 . Abhandlung von $\mathrm{Goltz}^{1}$ ) über die Verrichtungen des Grosshirns und den Hund ohne Grosshirn, an welchem trotz tiefen Blödsinnes, trotz Mangels an Gedächtniss und Ueberlegung, und obwohl kein Ausdruck von Freude oder von Neid und Missgunst an demselben zu beobachten war, Goltz doch noch eine Fülle von Lebensverrichtungen wahrnahm, die ihn über eine blosse Reflexmaschine erhaben erscheinen lassen. Gehbewegungen, Schlaf, Wachen, Hunger, Durst, Befriedigung nach der Fütterung, Zorn und Wuth, Knurren, Quieken, Bellen u. A. Auch die Arbeiten Flechsig's über die Projections- und Associations-Bahnen und Centren wurden erst lange später bekannt. Dennoch könnte ich mich mit dem Gehimanatomen Gall einerseits und mit dem Organologen Gall andererseits auch heute nicht anders auseinandersetzen, als ich es im Jahre 1882 gethan habe. Dafür sprechen auch die Bemerkungen, welche Flechsig in seiner Rede: "Gehirn und Seele" ${ }^{2}$ ) über Gall eingeflochten hat.

Es musste nun bei meiner Vertrautheit mit den Arbeiten Gall's im höchsten Grade verblüffend auf mich wirken, als ich in jüngster Zeit, nachdem ein Aushängebogen mit: "Soeben erschienen" von der renom-

1) Dieses Archiv Bd. 51 S. 570.

2) Zweite Anflage. Leipzig 1890. S. 12 u. 13. Auch Tigerstedt (Lehrbuch der Physiologie Bd. 2 S. 336, Leipzig 1898) wirft ein paar Streiflichter auf Gall und seine Organenlehre, um die neue Localisationslehre scharf von ihr zu scheiden, wäbrend P. J. Möbius (Schmidt's Jahrbücher der gesammten Medicin Bd. 262 S. 264, Leipzig 1899) nach nur flüchtiger Berührung der Organologie Gall's Verdienste um die Hirnanatomie klar zu legen sucht. 
mirten Berliner Verlagsbuchhandlung August Hirschwald vorausgegangen war, aus der Buchhandlung ein Heft $^{1}$ ) von einem Specialisten für Psychiatrie und Nervenkrankheiten in London erhielt, in welchem auch das Folgende zu lesen steht ${ }^{2}$ ): „Doch alle insgesammt" (es scheinen, da ganz unmittelbar vorher von Exner die Rede ist, die Physiologen gemeint zu sein) „bewusst oder unbewusst haben sie ein solches Vorurtheil gegen Gall, dass noch kein Menseh auf den Gedanken gekommen ist, sich Gall's Meisterwerk: Anatomie et Physiologie du Système nerveux en général et du cerveau en particulier, 4 Foliobände ${ }^{3}$ ) nebst einem Atlas von $100 \mathrm{Kupferstichen,} \mathrm{ein} \mathrm{Werk,} \mathrm{wie}$ kein zweites in diesem Jahrhundert ersehienen ist und das dem G all ein Vermögen kostete, auch nur anzusehen." Und weiter heisst es in demselben Hefte $^{4}$ ), nachdem ausgesprochen wurde, dass die anatomischen Funde Gall's für viele eine überraschende Neuigkeit sein werden: "Das ist der Gall, den ich kenne, den ich entdeckt habe und hiermit der heutigen Gelehrtenwelt vorführe. Der andere Gall, über den sich so viele ereifern, dem so viele das phrenologische System und jeden Unsinn, der in einem Handbuche oder Katechismus über Phrenologie steht, zuschreiben, existirt nur in der Einbildungskraft der Verfasser selbst."

Das ist doch starker Tabak fin de siècle:

\author{
"Wer ist der Segler, \\ Der Unheil bringt, \\ Dess Ruf wie aus anderer \\ Welt erklingt?" \\ Julius Wolf: Der filegende Holländer.
}

Wir wollen uns mit dem Truggewebe und Citatengewirre dieses fliegenden Holländers nicht des Näheren beschäftigen.

Aber einige Gall betreffende Irrthümer, wenn wir es so nennen sollen, möchte ich beleuchten.

1. Gall hat nicht schon vor Broca das Sprachcentrum entdeckt. Wer auf Tafel IV von Gall in der mit XV (entsprechend Sprachsinn) bezeichneten Hirnwindung die Reil'sche Insel sieht

1) Die Localisation der psychischen Thätigkeiten im Gehirn von med. Dr. Bernard Holländer. Berlin 1900. 32 Seiten.

2) S. 8 und 9.

3) Es existirt eine Ausgabe in Folio und eine in Quart des citirten Werkes s. d. frühere Citat.

4) S. 28. 
(besagtes Heft S. 9) und ebenso in der auf Tafel $V$ mit XV bezeichneten Windung und in Tafel XIII in der mit XV bezeichneten Stelle einen Querschnitt durch die Insel, hat keine Ahnung davon, welcher Hirntheil als Reil'sche Insel bezeichnet wird. Aber auch mit der dritten Frontalwindung stimmt die von Gall mit XV bezeichnete Stelle nicht überein, sondern fällt weit davon nach innen gegen die Medianebene hin.

2. Bemerke ich, dass Gall, wie schon gesagt, bei der Zergliederung des Gehirns zwar von der grauen Substanz ausgegangen ist und aus derselben alle Nerven entspringen lässt (besagtes Heft S. 28). Wenn man aber darum den Anschein erweckt, dass Gall eine geläuterte Anschauung über das Wesen der grauen Substanz gehabt habe, dann verbreitet man Irrthümer.

Nachdem Gall in dem früher schon citirten Memoire ${ }^{1}$ ) die Ausbreitung der grauen Substanz im Hirn und Rückenmark angegeben hat, fährt er fort: „Man begegnet ihr neben allen Nervenursprüngen, manchmal begleitet sie die Nerven in deutlich sichtbarer Weise in ihrem Verlaufe; man findet sie endlich in allen Nervenausbreitungen, z. B. in der Haut, wo diese den Namen des Malpighi'schen Schleimnetzes trägt; sie bildet die fast flüssige Sulze des Labyrinths, einen Theil der Schleimhaut in den Nasengängen, die Oberfläche der Retina etc.", und auf S. 138 des Memoires sagt Gall mit Bezug auf das Verhältniss von grauer und weisser Substanz: „diese Wabrheiten werden noch auffallender, wenn man die Gesetze der Vegetation mit jenen des Nervensystems zusammenstellt, weil man dann sieht, dass die Natur denselben Gang bei der Organisation des Pflanzenreiches einhält wie bei dem des Thierreiches", und weiter: „Mit vielem Vergnügen haben wir darum dieselben Gesetze festgestellt gefunden in der Metamorphose der Pflanzen von dem berühmten Goethe und in den Essais sur l'organisation des plantes von Herrn Aubert du Petit-Thuars". Hier erlaubt sich Gall offenbar auf ganz oberflächliche Kenntnisse hin eine Zusammenwerfung ganz verschiedener Dinge, auf die wir noch zurückkommen werden. Was will aber Gall daraus herleiten? nichts, als dass die graue Substanz des Nervensystems einen ähnlichen Nährboden für die Nervenfasern abgeben soll, wie ihn die Samenlappen und das Cambium für die Pflanzenfasern nach seiner Meinung bilden sollen.

1) Recherches sur le système nerveux en général etc. Paris 1809. p. 66 . E. Pfltig ex, Archiv für Physiologie. Bd. 79. 
Diese Ableitung kehrt auch im grossen Werke $^{1}$ ) wieder, nur sind dort Goethe und du Petit-Thuars nicht mehr eitirt. Solcher Wirrwarr findet sich also bei Gall über die graue Substanz.

3. Der letzte Punkt, welchen ich besprechen will, ist, dass es in dem besagten Hefte heisst, dass sich diejenigen ein Armuthszeugniss ausstellen, welche Gall für den Phrenologen halten; „denn hätten sie Gall's Meisterwerk: Anatomie et Physiologie du Système nerveux en général et du cerveau en particulier auch nur angesehen, so würden sie im dritten Band eine besondere Einleitung gesehen haben über Dr. Spurzheim, den Gehilfen und Mitarbeiter Gall's, der seinem Meister davongelaufen und ohne dessen Zustimmung ein phrenologisches System gegründet hat, dessen Fehler Gall hervorhebt, ein System, an das er nicht glauben will, weil seine eigenen Beobachtungen noch nicht vervollständigt und verificirt sind. Gall will Kopfformen nur da vergleichen, wo Untersuchung des Gehirns nicht möglich ist, wie bei lebenden Menschen."

Thatsache ist, dass Spurzheim sich von Gall trennte und dass Gall uber Spurzheim 's ${ }^{2}$ ) Versuch, die Sinne, Talente, Triebe, Begierde;, Leidenschaften etc. in ein besseres System zu bringen, und über Spurzheim's Erfolge in England, wo für die Organenlehre der Name Phrenologie aufkam, sehr aufgeregt war. So lästert er in einem Briefe ${ }^{3}$ ) vom 21. Februar 1827 an einen Freund in Wien diejenigen, welche seine Lehre "wahrscheinlich aus Spurzheim's verhunztem Werke kennen gelernt" haben. Wer aber Gall und Spurzheim kennt, wird zugeben müssen, dass sich der eine als Organologe und der andere als Phrenologe in gleich luftigen Speculationen ergehen.

Wenn aber Jemand die Secession Spurzheim's dazu benutzt, um Gall den Anstrich eines ernsten Forschers in der Localisation der psychischen Thätigkeiten im Gehirn zu geben und Spurzheim allein in die phrenologisehe Tinte zu bringen, so ist das ein wahrhaft aberwitziger Einfall.

1) Vol. 1. Paris 1810. p. 242 u. f.

2) Essai philosophique sur la nature morale et intellectuelle de l'homm?. Paris 1820. Wer sich für Spurzheim's zahlreiche englische Publicationen interessirt, findet dieselben in Index catalog. of the Library of the Surg.-gen. Off. U. S. Army vol. 13 p. 535 u. f. 1892.

3) In meinem Besitze befindlicher Briefwechsel zwischen Gall einerseits und Andreas Streicher und dessen Frau andererseits. 
In dieser Tinte steckten sie beide schon lange vor der Secession. Als sie das schon mehr erwähnte Memoire dem französischen Institut überreichten, wurden Tenon, Portal, Sabatier, Pinel und $\mathrm{Cuvier}$ als Berichterstatter gewählt. Der von $\mathrm{Cuvier}$ verfasste Bericht ${ }^{1}$ ) betont des Oeftern, dass die Commissäre mit den organologischen Speculationen der Autoren nichts zu thun baben wollen, sie halten nur über die anatomischen Arbeiten strenges Gericht und suchen ernüchternd auf die Speculationen zu wirken.

Nachdem in dem Berichte angeführt ist, dass dem Hirn dreierlei Verrichtungen zukommen: Sinneseindrücke aufzunehmen, dem Geist zu übertragen, die Eindrücke aufzubewahren und zu reproduciren, wenn ihrer der Geist bedarf und sie durch Ideen-Association erweckt werden, und die Gebote des Willens auf die Muskeln auszusenden, folgt die merkwürdige an du Bois-Reymond's Ignorabimus gemahnende Stelle: "diese drei Verrichtungen setzen aber den auf ewig unbegreiflichen wechselseitigen Einfluss der theilbaren Materie und des untheilbaren Ich voraus, die unüberschreitbare Kluft in unserem Ideensystem und den ewigen Stein des Anstosses aller Philosophien. Wir begreifen nicht nur nicht und werden nie begreifen, wie unser Geist die Eindrücke des Gehirns vernehmen und dadurch Bilder erhalten könne, sondern so fein auch immer unsere Untersuchungen sein mögen, so können wir doch die Spuren dieser Eindrücke niemals auffinden, und ihre Beschaffenheit bleibt uns immer unbekannt, obschon uns die Erscheinungen, welche im hohen Alter und in manchen Krankheiten statt haben, weder an ihrer Gegenwart noch an ihrem Sitze zweifeln lassen."

Welche Erlebnisse hatte aber Gall im Jahre 1809, als er mit Spurzheim das Memoire überreichte, als Organologe und Schädellehrer schon hinter sich?

Greifen wir nur eine Episode aus seinem 1805 beginnenden und bis zu seiner Niederlassung in Paris im Jahre 1808 dauernden Wanderleben heraus, und zwar die interessanteste.

Fr hielt wie in zahlreichen anderen Städten Deutschlands Vorlesungen über seine Lehre im Jahre 1805 auch in Halle, und dort traf er mit Goethe zusammen. Gall ${ }^{2}$ ) schrieb darüber an Streicher: "Als ich nach Halle kam, wartete schon Goethe auf mich; er war

1) Paris und Strassburg 1809.

2) Citirter Briefwechsel. 
in der Absicht dahin gereist, obschon er sich sehr übel befand. Er war mein eifrigster Zuhörer, und diese Ehre wurde mir sehr beneidet." Goethe war aber nur zu dem Besuche von F. A. Wolf nach Halle gereist, wie er selbst in den Annalen erzählt, und benutzte nur die Gelegenheit, Gall zu hören, was ihm allerdings willkommen war. Gall trat, wie uns Steffens ${ }^{1}$ ) berichtet hat, im grossen Saale eines Gasthofes auf, umgeben von seinem ganzen Apparate. Sein Vortrag floss leicht dahin. Einseitige sinnliche Wahrnehmungen, die er mit einem gewissen Scharfblicke für seine Zweeke herausfand und in deren Combination er ein grosses Talent zeigte, waren ihm sichere Quellen des Erkennens. Niemand wurde durch Zweifel irgend welcher Art so wenig gestört als Gall. Seine Zuversicht imponirte und überraschte im ersten Moment. Die Schädel berüchtigter Diebe verglich er mit denen der Elstern und Raben, die gefährlicher Mörder mit denen der Tiger und Löwen. Bedeutende Männer, F. A. Wolf, Schleiermacher, Fr. L.Froriep, Loder, Reil, Steffens, A. W. Niemayer, Reichardt u. A., sassen im Auditorium, und der bedeutendste von Allen mit stiller Aufmerksamkeit und gebietender Ruhe, in den edlen Gesichtszügen nur das steigende Interesse an der Entwicklung des Vortrages ansgedrückt, Goethe. Was Goethe in Gall's Vortrag besonders würdigte, hat er uns wieder selbst gesagt: „Ich war gewohnt, das Gehirn von der vergleichenden Anatomie her zu betrachten, wo es schon dem Auge kein Geheimniss bleibt, dass die verschiedenen Sinne als Zweige des Rückenmarks ausfliessen und erst einfach und einzeln zu erkennen, nach und nach aber schwerer zu beobachten sind, bis allmälig die angeschwollene Masse Unterschied und Ursprung verbirgt." Gall entsprach aber nicht ganz G o ethe's Erwartungen. Da Gall flüchtig. die Metamorphose der Pflanze berührte, sagte Go e th e später darüber : "Zu verwundern war es, dass er, ob er gleich die Analogie gefühlt haben musste, in der Folge nicht wieder darauf zurückkam, da doch diese Idee gar wohl durch sein ganzes Geschäft hätte walten können."

Wir baben schon früher ${ }^{2}$ ) gesehen, dass Gall die Metamorphose der Pflanze nicht ordentlich verstanden haben kann.

Als Gall in seinen Vorlesungen zu Halle zur Darstellung der

1) Drei Vorlesungen über Gall's Organenlehre. Halle 1805. Was ich erlebte. Bd. 4. Breslau 1842.

2) S. 307. 
Organe der verschiedenen Talente kam, berichtet uns Steffens weiter, sprach er zuerst von solchen Schädeln, welche keine Erhabenheit besonders ausgeprägt, wohl aber ein schönes bedeutendes Ebenmaass aller erkennen lassen. Und da scheute er sich nicht, als ein lehrreiches Beispiel eines solchen Gebildes den Kopf des grossen Dichters vorzuführen, der seine Vorträge mit seiner Gegenwart beehre. Alles sah auf Goethe, der ruhig blieb und ein kaum bemerkbares Missvergnügen mit ironischem Lächeln unterdrückte. Neben G o e the sassen der Componist Reichardt und Wolf, die Gall ebenso zur Demonstration des Tonsinnes und Sprachsinnes benutzte, wobei Wolf ergötzlicher Weise, als Gall nur erst an den Sprachsinn herangelangte, sich schon die Brille von der Nase genommen und den Kopf nach allen Seiten gewendet haben soll. Die schlagende Bestätigung seiner Lehre durch so auffallende Persönlichkeiten hatte aber mit grosser Gewalt auf Gall's Zuhörer gewirkt.

War also Gall der Schädellehrer oder macht ihn nur die Ignoranz der heutigen Physiologen dazu, wie das besagte Heft glauben machen will? Nein! Wir kennen Gall und wissen nach Gebühr über ihn zu urtheilen. $O b$ das von ihm gesammelte mannigfache casuistische Material noch einmal eine Verwerthung finden kann, darüber lässt sich nicht für alle Zukunft entscheiden.

Das abweisende und scharfe Urtheil, welches ich jetzt zum Schlusse über den im besagten Hefte gemachten Versuch einer Wiedererweckung des Organologen Gall fällen muss, will ich gar nicht direct aussprechen. Es ist so selbstverständlich, dass ich es jedem Leser überlassen kann, dieses Urtheil in die entsprechenden Worte zu kleiden. 